\title{
Complex Regional Pain Syndrome of Non-hemiplegic Upper Limb in a Stroke Patient: A Case Report
}

\author{
Ahry Lee, MD ${ }^{1}$, Youjin Jung, MEng ${ }^{1}$, Hee-Kyu Kwon, MD, PhD ${ }^{1}$, Sung-Bom Pyun, MD, PhD ${ }^{1,2}$
}

${ }^{1}$ Department of Physical Medicine and Rehabilitation, Korea University Anam Hospital, Seoul;

${ }^{2}$ Brain Convergence Research Center, Korea University Anam Hospital, Seoul, Korea

Complex regional pain syndrome (CRPS) type I in stroke patients is usually known to affect the hemiplegic upper limb. We report a case of CRPS presented in an ipsilesional arm of a 72-year-old female patient after an ischemic stroke at the left middle cerebral artery territory. Clinical signs such as painful range of motion and hyperalgesia of her left upper extremity, swollen left hand, and dystonic posture were suggestive of CRPS. A three-phase bone scintigraphy showed increased uptake in all phases in the ipsilesional arm. Diffusion tensor tractography showed significantly decreased fiber numbers of the corticospinal tract and the spinothalamic tract in both unaffected and affected hemispheres. Pain and range of motion of the left arm of the patient improved after oral steroids with a starting dose of $50 \mathrm{mg} /$ day.

Keywords Complex regional pain syndromes, Stroke, Diffusion tensor imaging

\section{INTRODUCTION}

Complex regional pain syndrome (CRPS) is a syndrome showing regional pain accompanied with vasomotor, sudomotor, sensory, motor, and/or trophic changes. It is a not a rare complication in stroke patients. It is usually known to affect the hemiplegic upper limb (UL). However, to the best of our knowledge, there has been no report of CRPS type I (CRPS-1) in the non-hemiplegic limb of stroke patients. Here we report a case of CRPS-1 in the non-hemiplegic UL after a left middle cerebral artery infarction.

\section{CASE REPORT}

A 72-year-old right-handed woman complained sudden right sided weakness and aphasia. She was diagnosed with an infarction of the left middle cerebral arterial territory (Fig. 1A) and treated with intravenous tissue plasminogen activators. A few hours later, the infarction 

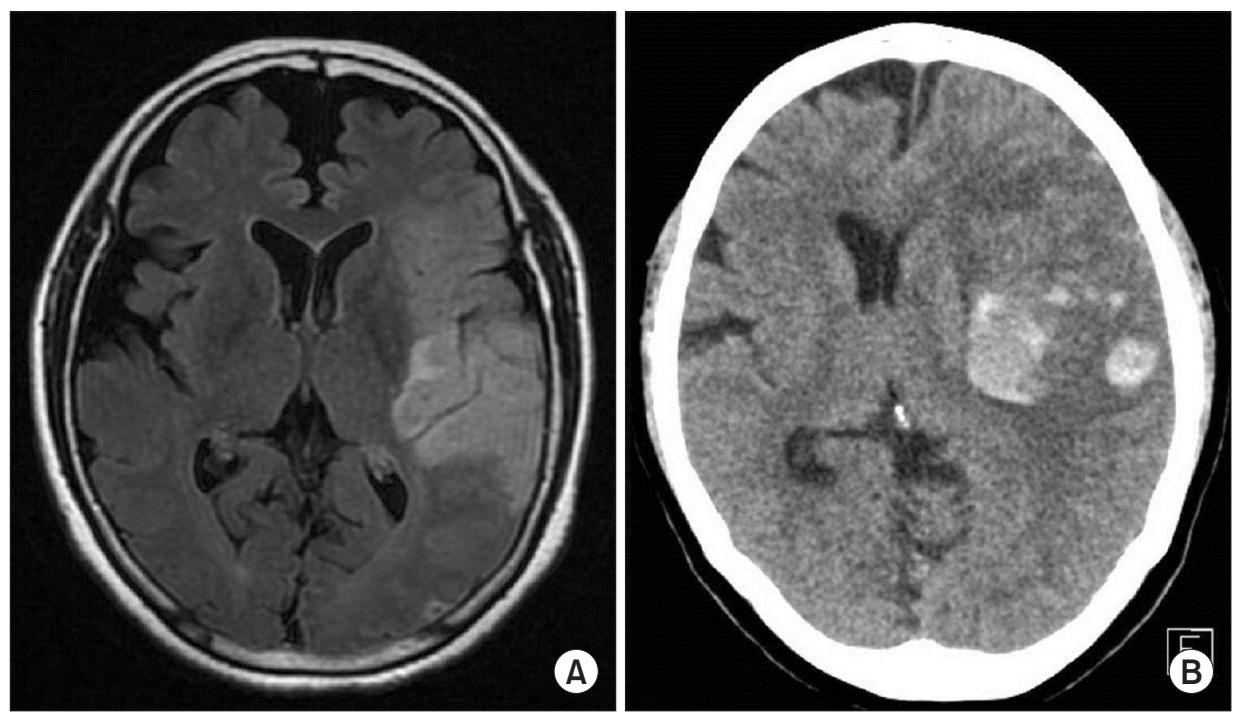

Fig. 1. (A) Brain magnetic resonance imaging (T2 FLAIR image, axial view) showing infarction of the left middle cerebral arterial territory. (B) Brain computed tomography image 1 day after infarction showing an acute hemorrhagic transformation resulting in midline shifting.

had undergone hemorrhagic transformation, resulting in midline shifting (Fig. 1B). As the midline shift secondary to mass effect and brain swelling progressed, the patient received decompressive craniectomy on the next day.

Her past medical history included hypertension and atrial fibrillation for which she was taking medication including aspirin. She did not have history of prior cerebrovascular attack, head injury, or trauma.

At 2 months after onset, she was referred to receive rehabilitation for her right sided flaccid hemiplegia, cognitive impairment, and global aphasia. She was bed-ridden and unable to follow 1-step command. Her right limbs were flaccid. However, her left limbs showed spontaneous movements. They were able to move against gravity. Spasticity of the left elbow flexor muscles was grade 2 according to the Modified Ashworth Scale.

Her left UL showed sustained contraction, resulting in a dystonic posture with a flexed elbow, wrist, and fingers (Fig. 2). The patient's frown face suggested pain on her left UL when it passively moved. Painful ranges of motion (ROM) and hyperalgesia of the left hand, wrist, and shoulder areas were present. Her left hand was swollen mildly without trophic changes. She had no definite history of trauma or nerve injury in her ipsilesional UL. All blood tests for infections, deep vein thrombosis, liver, and renal disease were within normal ranges. There were no epileptiform discharges on electroencephalography or any structural lesion of the contralesional right cerebral hemisphere. These clinical findings were sufficient to fulfill the revised International Association for the Study

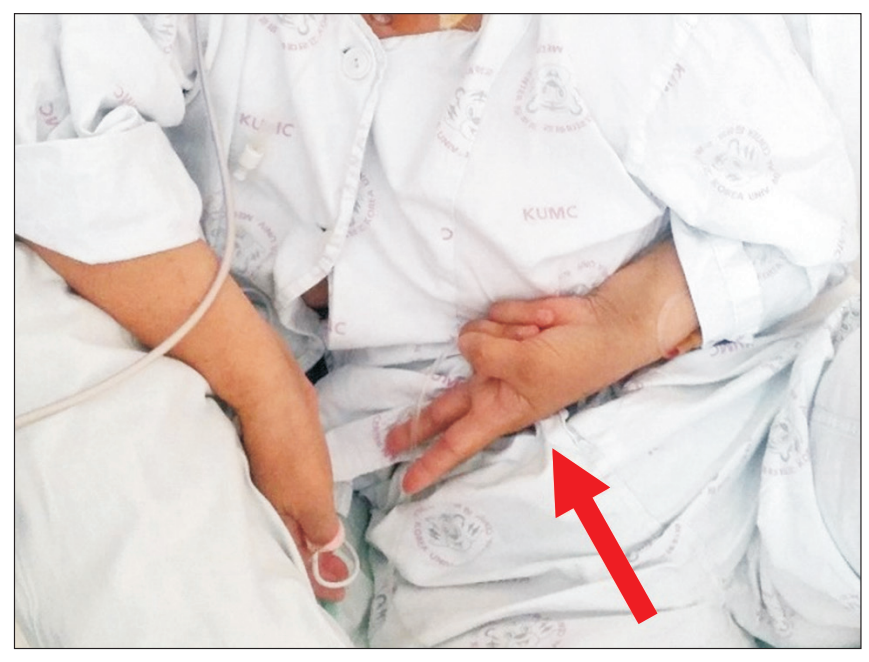

Fig. 2. Dystonia like posture of the left upper limb. Note fingers of the left hand forming a ' $\mathrm{V}$ ' shape (arrow).

of Pain (IASP) diagnostic criteria (also called the Budapest criteria suggested by the Budapest IASP consensus group in 2003 [1]) for CRPS. After CRPS was clinically suspected, a three-phase bone scintigraphy was performed, resulting in higher perfusion and blood pools in the left UL than those in the right (Fig. 3A, 3B). Delayed images showed diffusely increased radionuclide uptakes in the left UL (Fig. 3C). These findings were compatible with CRPS.

To investigate underlying neuroanatomical mechanisms, diffusion tensor imaging (DTI) was performed using a 3.0-T magnetic resonance imaging (MAGNETOM Trio a Tim system; Siemens, Erlangen, Germany) 
to evaluate corticospinal tract (CST) and spinothalamic tract (STT) - parameters: matrix $=128 \times 128$, field of view $=230.4 \times 230.4 \mathrm{~mm}^{2}$, voxel size $(\mathrm{x}, \mathrm{y}, \mathrm{z})=1.8 \times 1.8 \times 2$
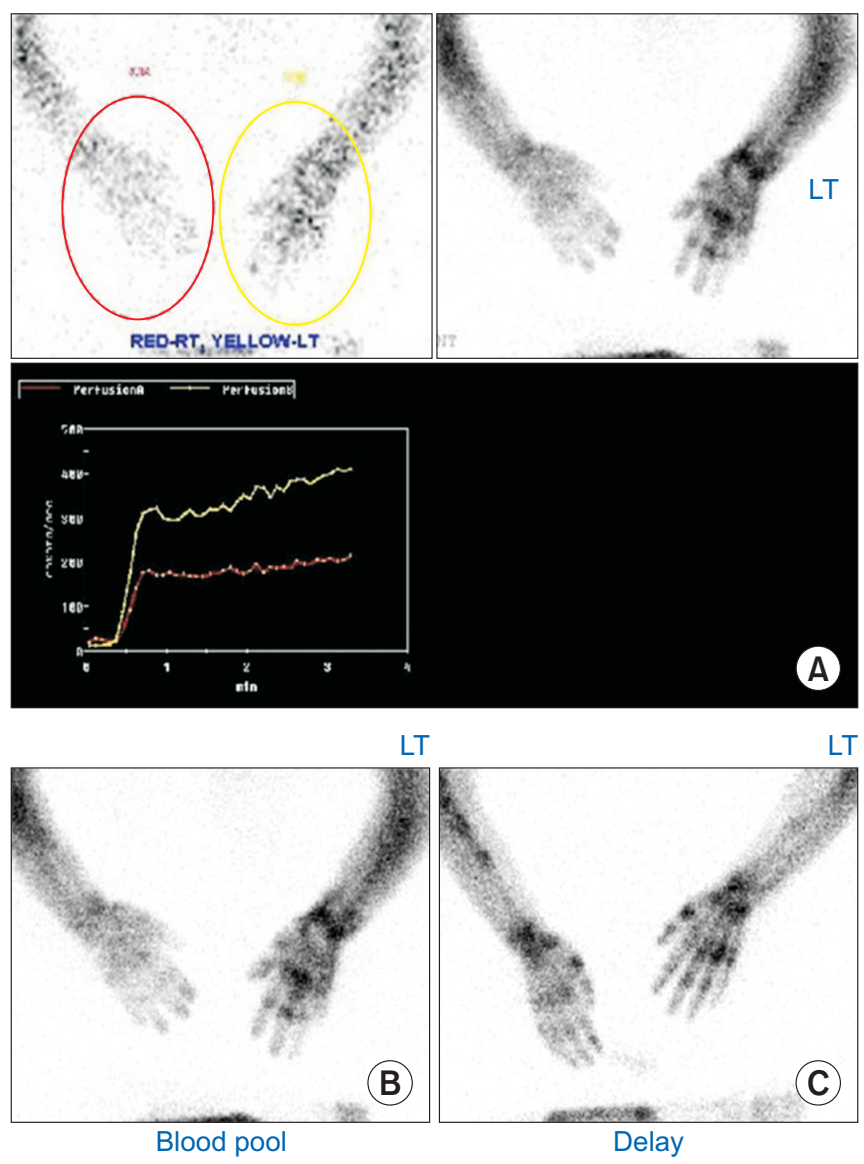

Fig. 3. Three-phase bone scintigraphy of clinically suspected complex regional pain syndrome (CRPS) of a nonhemiplegic upper limb in the patient with left cerebral infarction. (A) Perfusion phase. Perfusion to the left hand was higher than that to the right hand. (B) Blood pool phase. Blood pool to the left hand was higher than that to the right hand. (C) Delayed phase. Diffusely increased radionuclide uptakes in the left upper extremity. $\mathrm{mm}^{3}, \mathrm{TE}=89 \mathrm{~ms}, \mathrm{TR}=9,100 \mathrm{~ms}, \mathrm{~b}=1,000 \mathrm{~s} / \mathrm{mm}^{2}$, slice thickness $=2.3 \mathrm{~mm}$, flip angle $=90^{\circ}$, and directions $=20$. Fiber tracking of CST and STT was done using DTI Studio software (V3.0.2; free software from the Department of Radiology, Johns Hopkins University, Baltimore, MD, USA) (minimum fractional anisotropy $=0.1$, and tractturning angle $=70^{\circ}$ ). CST or STT was not visualized using two regions of interest (ROIs) method (CST, the anterior mid-pons and the primary sensorimotor cortex; STT, the posterolateral medulla and the primary sensory cortex). Additional tract reconstruction was performed manually placing one seed ROI on the anterior mid-pons and the posterolateral medulla for CST and STT, respectively. Fiber numbers of CST and STT were significantly decreased in both unaffected and affected hemispheres (Table 1, Fig. 4).

Oral steroids were used $50 \mathrm{mg} /$ day for 7 days. They were then tapered out over 9 days. Passive ROM exercise was applied gently on her left UL. Limited ROM, swelling, and pain in the left UL improved after the treatment.

\section{DISCUSSION}

Our case was diagnosed as CRPS- 1 in a non-hemiplegic UL after an ischemic stroke. There has been no such report to the best of our knowledge. The pathophysiology of CRPS is not well known yet. Inflammation, nociceptive sensitization, vasomotor dysfunction, and maladaptive neuroplasticity have been proposed [2]. Although the central mechanism of post-stroke CRPS is still unclear, microtraumas to the hemiplegic shoulder and deterioration of biomechanical factors might play significant roles in the genesis of CRPS in stroke patients [3]. Although major injuries were not reported in our case, minor soft tissue or peripheral nerve trauma might have resulted from unrecognized shoulder traction or nerve compres-

Table 1. Parameters of diffusion tensor tractography of CST and STT in each hemisphere

\begin{tabular}{lccccc}
\hline & \multicolumn{2}{c}{ CST } & \multicolumn{2}{c}{ STT } \\
\cline { 2 - 3 } \cline { 5 - 6 } Number of fibers & Left (affected) & Right (unaffected) & & Left (affected) & Right (unaffected) \\
\hline FA & 12 & 18 & 144 & 54 \\
AD & 0.5315 & 0.6189 & & 0.4597 & 0.4938 \\
RD & 1.083 & 1.113 & 1.630 & 1.546 \\
MD & 0.491 & 0.410 & 0.849 & 0.753 \\
\hline
\end{tabular}

CST, corticospinal tract; STT, spinothalamic tract; FA, fractional anisotropy; AD, axial diffusivity $\left(10^{-3} \mathrm{~mm}^{2} / \mathrm{s}\right)$; RD, radial diffusivity $\left(10^{-3} \mathrm{~mm}^{2} / \mathrm{s}\right)$; MD, mean diffusivity $\left(10^{-3} \mathrm{~mm}^{2} / \mathrm{s}\right)$. 

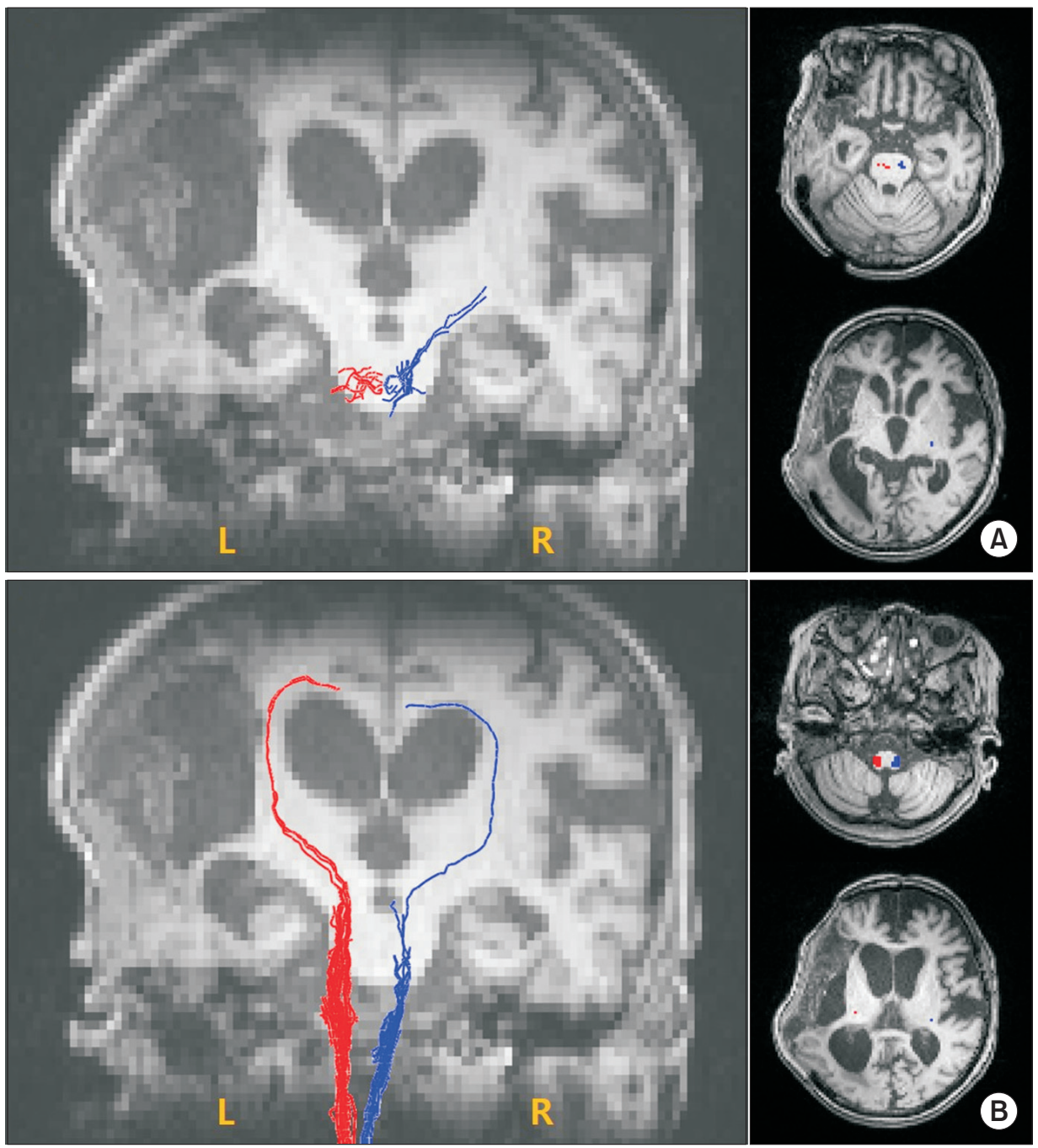

Fig. 4. Tractography for the corticospinal tract (A) and spinothalamic tract (B) showing significant volume reduction in both hemispheres.

sion. The patient's severe right hemiplegia and poor cooperation due to global aphasia might have led to repeated minor traumas unrecognized by caregivers or clinicians.

Neuroplastic change of the central nervous system is also suggested as a possible pathophysiologic mechanism of CRPS. It includes supraspinal involvement and spinal neuronal sensitization characterized by hyperexcitability of excitatory neurons and disinhibition of nociceptive neurons [2]. Functional neuroimaging studies have demonstrated that maladaptive cortical reorganization and dysfunctions of primary motor and somatosensory cortices are associated with CRPS [4]. Some studies have suggested that subcortical structures may also contribute to clinical presentation of CRPS. A previous study has shown that CRPS patients show reorganizations of white matter connectivity with reduced fractional anisotropy in the left cingulum-callosal bundle [5]. Another study has suggested that basal ganglia dysfunctions might be a possible pathophysiologic cause of CRPS [6]. One study has found that an absent median somatosensory evoked potential is a primary predictor of post-stroke CRPS [7]. Therefore, it has been proposed that the somatosensory pathway is involved in the genesis of CRPS [7]. In our case, diffusion tensor tractography showed markedly decreased fiber numbers of CST and STT not only in the affected hemisphere, but also in the unaffected left hemisphere. This might be due to hemorrhagic transformation of the infarction which causes midline shifting with subfalcine herniation [8]. No known study has successfully investigated the association between an injury of CST or STT and CRPS in stroke patient, although some studies have proposed that injury of the STT is a prerequisite for post-stroke central pain [9]. Considering that CRPS and 
central pain after stroke show considerable overlapping clinical presentations, injury of STT or CST might play some roles in the development of CRPS.

Considering the role of central nervous system in the pathogenesis of CRPS [2,4-7] and the hypothesis that peripheral lesion might initiate a vicious cycle of pain in some post-stroke CRPS patients [10], destructive changes of the motor and sensory system in the contralesional hemisphere might be the underlying neuroanatomical mechanism in our case. Unrecognized minor peripheral lesions of the non-hemiplegic UL might trigger ipsilesional CRPS-1.

In summary, we report a rare case of CRPS-1 in an unaffected upper extremity. CRPS was easily diagnosed by a three-phase bone scan in addition to clinical symptoms and signs. The patient showed very good response to oral corticosteroid therapy. Therefore, CRPS should be taken into consideration if a stroke patient complains severe regional pain accompanied by vasomotor or sudomotor symptoms in an unaffected limb.

\section{CONFLICT OF INTEREST}

No potential conflict of interest relevant to this article was reported.

\section{ACKNOWLEDGMENTS}

This work was supported by a grant (No. 2016R1A2B400 9206) of the National Research Foundation of Korea (NRF) funded by the Korea government.

\section{REFERENCES}

1. Norman Harden R, Bruehl SP. Diagnostic criteria: the statistical derivation of the four criterion factors.
In: Wilson PR, Stanton-Hicks M, Harden RN, editors. CRPS: current diagnosis and therapy. 1st ed. Seattle: IASP Press; 2005. p. 45-58.

2. Marinus J, Moseley GL, Birklein F, Baron R, Maihofner C, Kingery WS, et al. Clinical features and pathophysiology of complex regional pain syndrome. Lancet Neurol 2011;10:637-48.

3. Chae J. Poststroke complex regional pain syndrome. Top Stroke Rehabil 2010;17:151-62.

4. Schwenkreis P, Maier C, Tegenthoff M. Functional imaging of central nervous system involvement in complex regional pain syndrome. AJNR Am J Neuroradiol 2009;30:1279-84.

5. Geha PY, Baliki MN, Harden RN, Bauer WR, Parrish TB, Apkarian AV. The brain in chronic CRPS pain: abnormal gray-white matter interactions in emotional and autonomic regions. Neuron 2008;60:570-81.

6. Azqueta-Gavaldon M, Schulte-Gocking H, Storz C, Azad S, Reiners A, Borsook D, et al. Basal ganglia dysfunction in complex regional pain syndrome: a valid hypothesis? Eur J Pain 2017;21:415-24.

7. Han EY, Jung HY, Kim MO. Absent median somatosensory evoked potential is a predictor of type I complex regional pain syndrome after stroke. Disabil Rehabil 2014;36:1080-4.

8. Hong JH, Kim SH, Kim OL, Byun WM, Jang SH. Neural tract injuries by brain herniations after head trauma. J Head Trauma Rehabil 2012;27:154-8.

9. Hong JH, Bai DS, Jeong JY, Choi BY, Chang CH, Kim $\mathrm{SH}$, et al. Injury of the spino-thalamo-cortical pathway is necessary for central post-stroke pain. Eur Neurol 2010;64:163-8.

10. Wasner G, Schattschneider J, Binder A, Baron R. Complex regional pain syndrome: diagnostic, mechanisms, CNS involvement and therapy. Spinal Cord 2003;41:61-75. 